\title{
Levels of leptin and IL-6 in lungs and blood are associated with the severity of chronic obstructive pulmonary disease in patients and rat models
}

\author{
RUI LIANG ${ }^{1}$, WEI ZHANG ${ }^{2}$ and YA-MEI SONG ${ }^{2}$ \\ ${ }^{1}$ Department of Respiratory Medicine, The Fourth Affiliated Hospital of Harbin Medical University, Harbin, \\ Heilongjiang 150001; ${ }^{2}$ Department of Respiratory Medicine, The First Affiliated Hospital \\ of Harbin Medical University, Harbin, Heilongjiang 150001, P.R. China
}

Received November 6, 2012; Accepted February 25, 2013

DOI: $10.3892 / \mathrm{mmr} .2013 .1377$

\begin{abstract}
The aim of the present study was to compare leptin and interleukin (IL)-6 expression in patients and rat models with chronic obstructive pulmonary disease (COPD). Leptin and IL-6 levels were determined in patients with an acute exacerbation of COPD (AECOPD), stable COPD and in healthy controls. Rat models of COPD were developed, histological and immunohistochemical analyses were performed and leptin and IL-6 levels were determined. Leptin and IL-6 levels in the serum and sputum were higher in patients with AECOPD compared with stable COPD and control patients. In rats, leptin and IL- 6 were expressed in bronchial epithelial and inflammatory cells, while leptin expression was observed in alveolar cells and IL-6 expression in blood vessel cells only. Serum levels of leptin and IL-6 were significantly higher in COPD1 and COPD2 rats compared with the control rats, and were even higher in COPD1 rats than COPD2 rats. In conclusion, leptin and IL-6 levels were demonstrated to be associated with the severity of COPD.
\end{abstract}

\section{Introduction}

Chronic obstructive pulmonary disease (COPD) is a largely irreversible progressive lung disease and is one of the leading causes of death and disability worldwide (1-3). It is estimated that COPD may be the third leading cause of death in the world by $2020(3,4)$. COPD is a common disease with high morbidity and mortality rates in China and several other developing countries $(5,6)$. COPD is characterized by chronic inflammation of the airways, chronic airflow limitation and compromised

Correspondence to: Professor Wei Zhang, Department of Respiratory Medicine, The First Affiliated Hospital of Harbin Medical University, 23 Post Street, Nangang, Harbin, Heilongjiang 150001, P.R. China

E-mail: weizhanghmu@yahoo.cn

Key words: chronic obstructive pulmonary disease, interleukin-6, leptin immune function in the body $(7,8)$. Malnutrition develops in 25-65\% of COPD patients; COPD-associated malnutrition is attributed not only to the disease condition, but also to a number of endogenous factors, including leptin levels (9-12). Leptin is an adipocyte-derived hormone that is important in energy homeostasis; its role is to inform the brain of the volume of adipose tissue stored in the body $(13,14)$. Leptin is also a proinflammatory factor that promotes the release of other inflammatory factors and positively regulates the immune function by exerting protective effects (15). Therefore, leptin may be important in the occurrence and development of COPD. To clarify the pathogenesis of COPD, it may be useful to determine the leptin levels in biopsy sample tissues or blood and to evaluate the prognosis and treatment of COPD patients.

Thus, extensive investigation into the association of leptin and inflammatory factors with the pathological processes of COPD is required. In this study, the levels of leptin and inflammatory factor interleukin (IL)- 6 were determined in patients with an acute exacerbation of COPD (AECOPD) and stable COPD, and in rat models of acute COPD, which have the potential to significantly impact the future treatment strategies of COPD.

\section{Materials and methods}

Clinical study. A total of 51 outpatients and inpatients with COPD and 20 healthy subjects undergoing physical examinations in the Fourth Affiliated Hospital of Harbin Medical University were included in this study. Serum and sputum samples were collected from the subjects and radioimmunoassay (RIA) kits (Beijing Furui Biotech Co., Ltd., Beijing, China) were used to determine the levels of leptin and IL-6. Fasting venous blood was obtained to determine white blood cell (WBC) counts and serum albumin (ALB) levels. Lung function was assessed in each subject using spirometry (MS-IOS; Jaeger, Hoechberg, Germany); the forced expiratory volume in one second (FEV1) was recorded and spirometry was performed three times. Differences of $<10 \%$ between two measures of FEV1 were used as the final FEV1 result, the average was calculated and the FEV1 percentage (FEV1\%) was determined. 
Animal experiments. Eight-week-old male Sprague-Dawley (SD) specific pathogen-free rats weighing $200 \pm 20 \mathrm{~g}$ were purchased from the Experimental Animal Center of Norman Bethune University of Medical Science (Changchun, China). For tobacco smoke exposure, Daguang filter cigarettes were used (tar content, $15 \mathrm{mg}$; nicotine content, $1.1 \mathrm{mg}$; Kunming Hongta Tobacco Co., Ltd., Kunming, China). Enzyme-linked immunosorbent assay (ELISA) kits for leptin and IL-6 were purchased from Beijing Biosen Biotech Co., Ltd. (Beijing, China), RIA kits were from Beijing Furui Biotech Co., Ltd. (Beijing, China) and lipopolysaccharide (LPS) was from Harbin Hongbo Xinye Biotech Co., Ltd. (Harbin, China).

\section{Study groups}

Clinical study. A total of 51 inpatients and outpatients with COPD who were admitted to the Department of Respiratory Diseases in the Fourth Affiliated Hospital of Harbin Medical University between December 2010 and June 2011 were included in this study. There were 27 patients with AECOPD and 24 with stable COPD. All the patients were male, $<80$ years of age and had a normal body mass index (BMI). COPD was diagnosed according to the GOLD Guidelines for COPD (2008) and additional metabolic diseases were excluded. Support therapy was provided to inpatients and glucocorticoids were administered prior to the study. The control group included 20 healthy subjects who underwent physical examinations in our hospital. All the subjects were male, $<80$ years of age and had a normal BMI. A review of the medical histories, physical examinations and accessory examinations of patients excluded the presence of diabetes and organic diseases of the heart, brain, liver and kidney. Additionally, patients had no systemic infections during the last 2 months. During the study, all the subjects kept a routine diet. The present study was approved by the Ethics Committee on Human Research of our hospital and written informed consent was obtained from the patients.

Animal experiments. A total of 36 male SD rats were randomly allocated to 3 groups (12 rats/group); the healthy control, COPD1 and COPD2 groups. The animals were housed in the Animal Center of The Fourth Hospital of Harbin Medical University at $20 \pm 2^{\circ} \mathrm{C}$ with a humidity of $40-70 \%$ under a 12:12-h light-dark cycle. Animals were given ad libitum access to general rat chow and water. The passive smoking equipment was prepared as previously described (automatic combustion-supporting ashtray). Rats in the COPD1 group were intratracheally administered LPS $(200 \mu \mathrm{g})$ on days 1 and 14, and then exposed to $5 \%$ smoke for $2 \mathrm{~h}$ daily for 4 consecutive weeks (with the exception of days 1 and 14). Rats in the COPD2 group were exposed to $5 \%$ smoke for $2 \mathrm{~h}$ daily for 12 consecutive weeks. Rats in all three groups were housed in the same environment; however, rats in the control group were not exposed to smoke. All the experimental protocols were approved by the Animal Care and Use Committee of our Institute.

\section{Sample collection}

Human clinical study. Blood and sputum samples were collected, centrifuged and stored for future use. RIA kits were used to determine leptin and IL-6 levels in the serum and sputum. The fasting venous blood was used for WBC counts and ALB level determination. Lung function was also assessed in each subject.
Animal experiments. After the COPD model was established in the rats, they were intraperitoneally anesthetized with $25 \%$ urethane $(1,000 \mathrm{mg} / \mathrm{kg})$. Subsequently, $4 \mathrm{ml}$ of blood was collected from the abdominal aorta and centrifuged at 3,000 rpm for $15 \mathrm{~min}$. Serum was collected and stored in aliquots at $-70^{\circ} \mathrm{C}$ for later use. The lungs were harvested, fixed in formalin solution for 35 days, embedded and sectioned, followed by hematoxylin and eosin (H\&E) staining and the immunohistochemical analysis of leptin and IL-6. RIA kits were used to determine the serum leptin and IL-6 levels according to the manufacturer's instructions.

Immunohistochemical analysis. The following criteria were used for determining protein expression: At a high magnification, cells with yellow-brown granules in the cytoplasm and nucleus were evaluated as positive for a target protein. The PA800 computerized pathological image analyzer (Beckman Coulter, Miami, FL, USA) was used for analysis and bronchioles with a diameter of 100-200 $\mu \mathrm{m}$ with a surrounding alveolar area were selected under a light microscope (magnification, x400). Three fields were randomly selected and the positive signal optical density was determined.

Statistical analysis. SPSS version 17.0 software was used for statistical analysis. Results for quantitative variables are expressed as the mean \pm standard deviation and results for ranked data are presented as medians and interquartile ranges (possible outliers were determined using the Q test). Means among the different groups were compared using analysis of variance or the rank sum test. $\mathrm{P}<0.05$ was considered to indicate a statistically significant difference.

\section{Results}

Patient serum and sputum sample analysis. As shown in Fig. 1, the serum levels of leptin and IL-6 and WBC counts in the AECOPD patients were significantly higher compared with those in patients with stable COPD and healthy controls $(\mathrm{P}<0.05)$. These were only slightly increased in patients with stable COPD compared with healthy controls $(\mathrm{P}>0.05)$. In the control group, ALB levels and FEV1\% were markedly higher compared with those in the COPD groups $(\mathrm{P}<0.05)$, while they were significantly lower in the AECOPD group compared with stable COPD patients $(\mathrm{P}<0.05)$.

As shown in Fig. 2, the sputum levels of leptin and IL-6 in patients with AECOPD were significantly higher compared with those in stable COPD and healthy control patients $(\mathrm{P}<0.05)$. In the serum samples, leptin and IL-6 levels were slightly increased in patients with COPD compared with healthy control patients $(\mathrm{P}>0.05)$.

\section{Rat COPD models}

General status. In COPD rats, the rate of body weight gain was slower compared with that in control rats (Fig. 3). Additionally, the hair of COPD rats was lusterless and became yellow. These animals were largely unanimated, had reduced activities and flaring nares. Cyanosis, tachypnea and wheezing were also noted.

Lung pathology. Representative rat lung histology sections after H\&E staining are shown in Fig. 4Aa (control), 
A

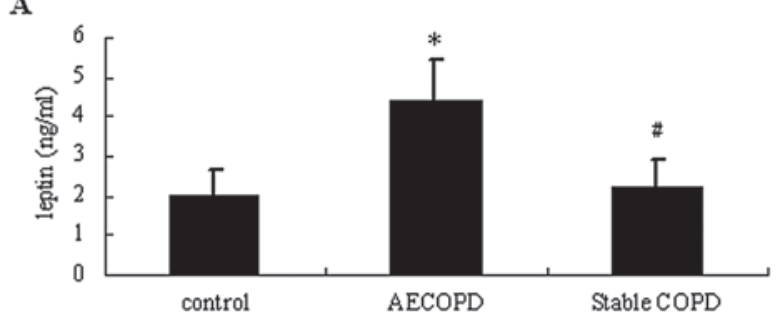

C

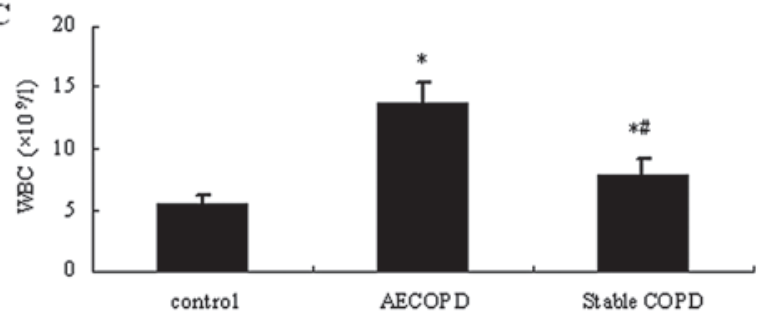

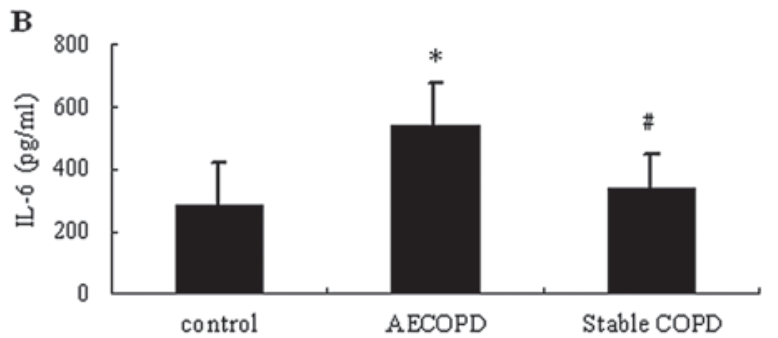

D

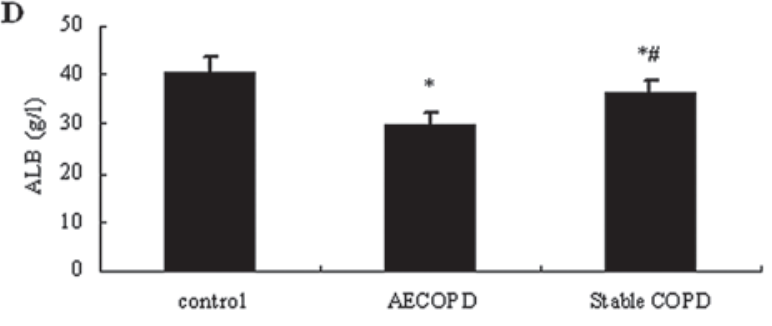

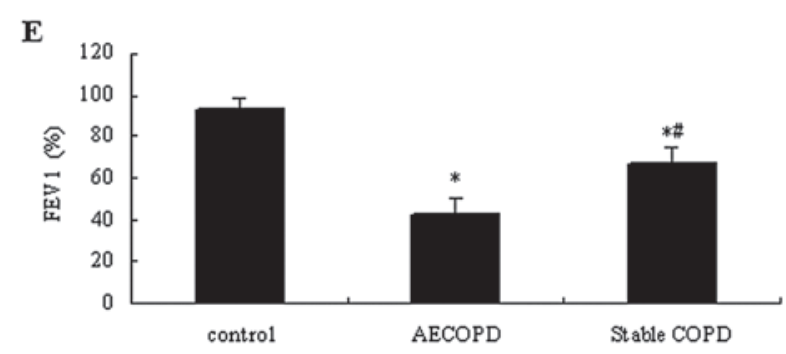

Figure 1. (A) Serum leptin and (B) IL-6 levels, (C) white blood cell (WBC) counts, (D) albumin (ALB) levels and (E) the forced expiratory volume in one second percentage (FEV1\%) in human subjects. Sample numbers of the control, AECOPD and stable COPD groups were 20, 27 and 24, respectively. The results are presented as the mean \pm standard deviation. ${ }^{\mathrm{P}}<0.05$ vs. the control group; ${ }^{\text {}} \mathrm{P}<0.05$ vs. the AECOPD group. COPD, chronic obstructive pulmonary disease; AECOPD, acute exacerbation COPD; IL-6, interleukin-6.
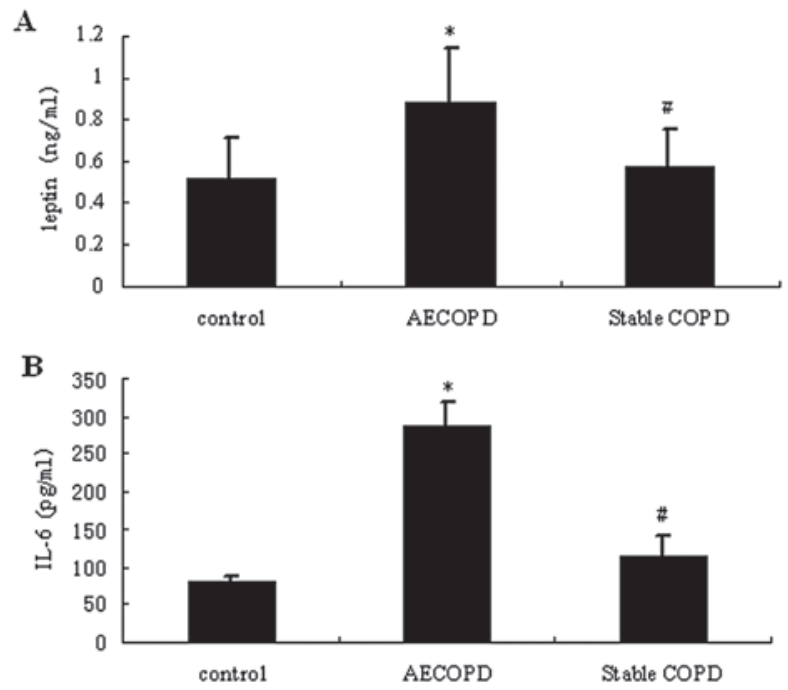

Figure 2. Sputum levels of (A) leptin and (B) IL-6 in human subjects. Sample numbers of the control, AECOPD and stable COPD groups were 20, 27 and 24, respectively. The results are presented as the mean \pm standard deviation. ${ }^{*} \mathrm{P}<0.05$ vs. the control group; ${ }^{\#} \mathrm{P}<0.05$ vs. the AECOPD group. COPD, chronic obstructive pulmonary disease; AECOPD, acute exacerbation COPD; IL-6, interleukin-6.

Fig. 4Ab (COPD1) and Fig. 4Ac (COPD2). With regard to the macroscopic assessment of the lungs, rats in the COPD groups had significantly larger lungs than rats in the control group.

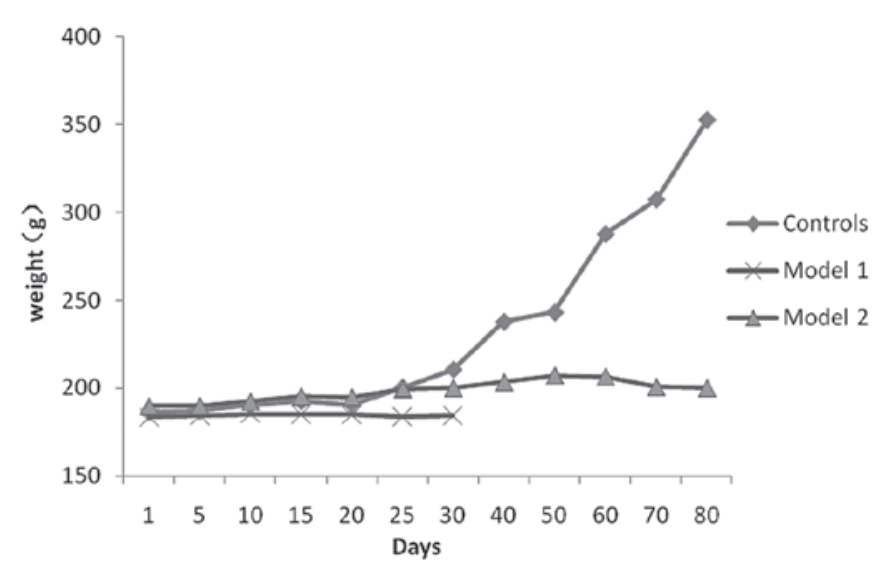

Figure 3. Changes in the mean body weight of rats $(n=12)$.

Additionally, the lungs of rats in the COPD groups were pale and had bullae, although hemorrhage and exudation were not observed.

In terms of the microscopic findings, the alveolar walls of COPD rats were partially thickened and large numbers of neutrophils, lymphocytes and mononuclear macrophages were observed around the trachea and blood vessels. Focal hemorrhage was noted and exudates were observed in the trachea. Furthermore, the alveolar walls were thin, those close to capsules were interrupted and several bullae were observed (emphysema). 

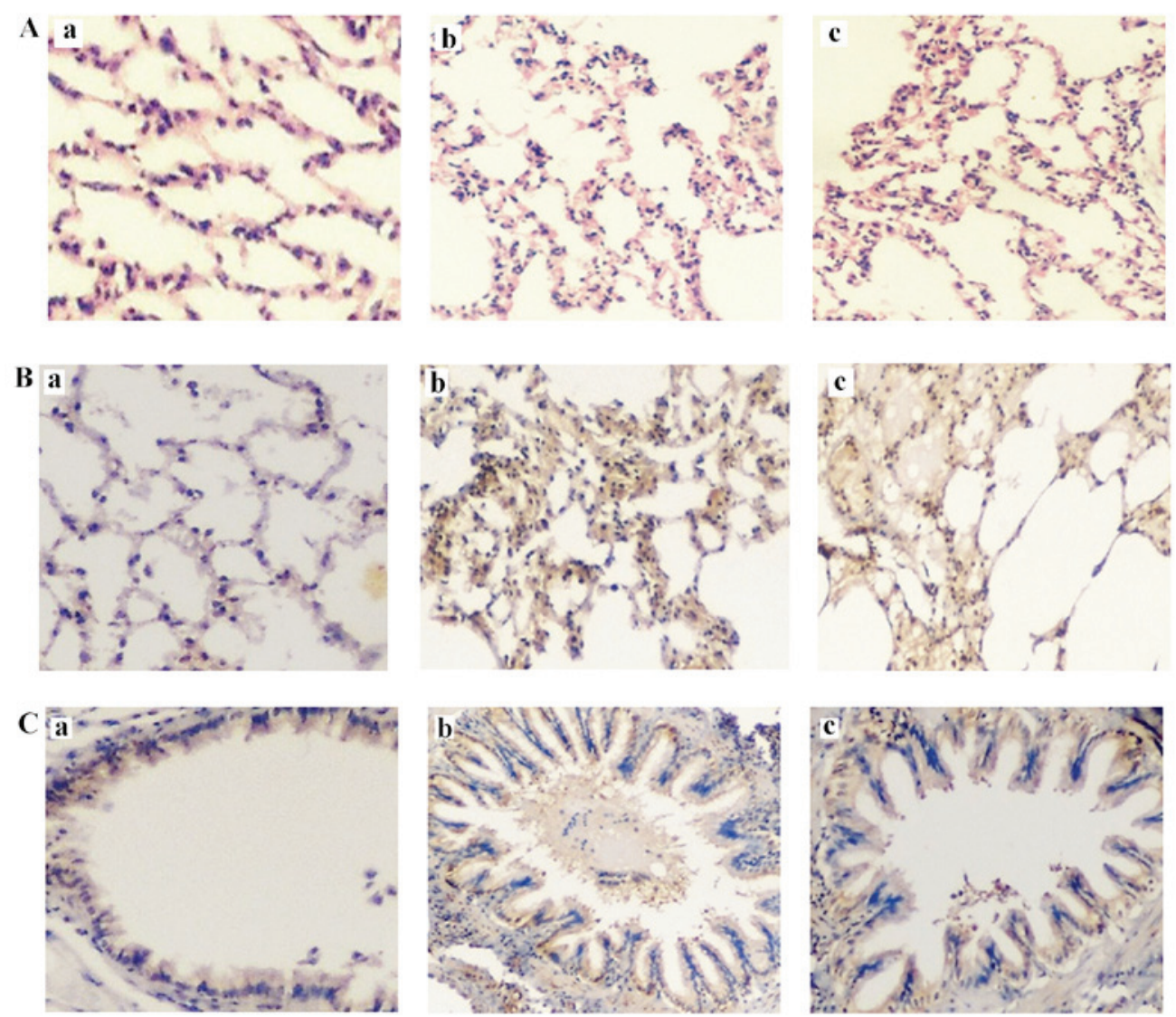

Figure 4. (A) Lung pathology and (B) leptin and (C) IL-6 expression in lung tissues. Tissue from (a) normal lungs and the (b) COPD1 and (c) COPD2 groups (magnification, x400). COPD, chronic obstructive pulmonary disease; IL-6, interleukin-6.

A

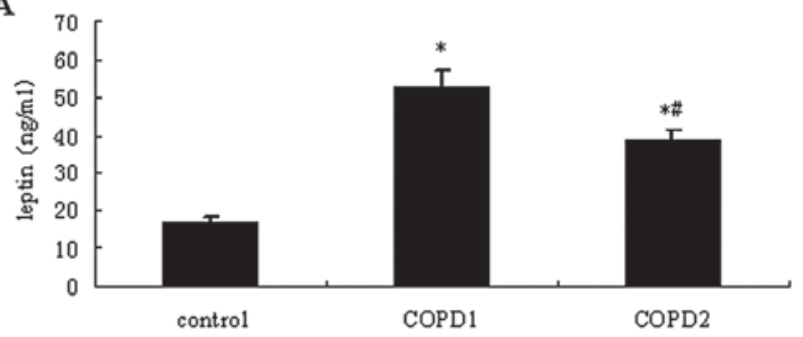

B

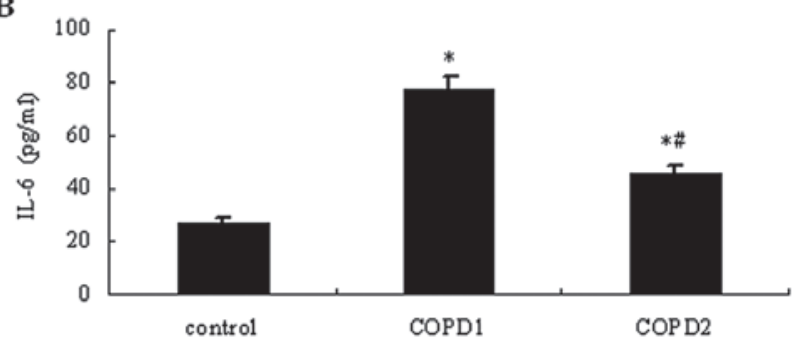

Figure 5. (A) Leptin and (B) IL-6 expression in the lungs of rats. The results are presented as the mean \pm standard deviation ( $\mathrm{n}=12$ ). ${ }^{*} \mathrm{P}<0.05$ vs. the control group; ${ }^{*} \mathrm{P}<0.05$ vs. the COPD1 group. COPD, chronic obstructive pulmonary disease; IL-6, interleukin-6.

Immunohistochemical analysis. Target protein expression in lung tissues was determined as described in Materials and methods. Representative rat lung histology sections following staining for leptin are shown in Fig. 4Ba (control), Fig. 4Bb (COPD1) and Fig. 4Bc (COPD2) and comparisons for leptin expression among the groups of rats are summarized in Fig. 5. Leptin expression was detected in bronchial epithelial cells, the alveolar wall and all the inflammatory cells. In the control group, a notably small fraction of bronchial epithelial cells were positive for leptin. In the COPD groups, leptin expression was significantly increased compared with that of the control group $(\mathrm{P}<0.05)$. Furthermore, a significant difference in leptin expression was identified between the COPD1 and COPD2 groups $(\mathrm{P}<0.05)$.
Representative rat lung histology sections following staining for IL-6 are shown in Fig. 4Ca (control), Fig. 4Cb (COPD1) and Fig. 4Cc (COPD2) and comparisons for IL-6 expression among the groups of rats are summarized in Fig. 5. A small number of bronchial epithelial and inflammatory cells in the alveolar wall expressed IL-6. IL-6 expression was also detected in blood vessels. In the COPD groups, IL-6 expression levels were markedly increased compared with those of the control group $(\mathrm{P}<0.05)$. A significant difference in IL-6 expression was also observed between the COPD1 and COPD2 groups $(\mathrm{P}<0.05)$.

Following COPD induction in the rats, blood was collected from the aorta and used to determine the leptin and IL-6 serum levels. As shown in Fig. 6, leptin and IL-6 serum levels 

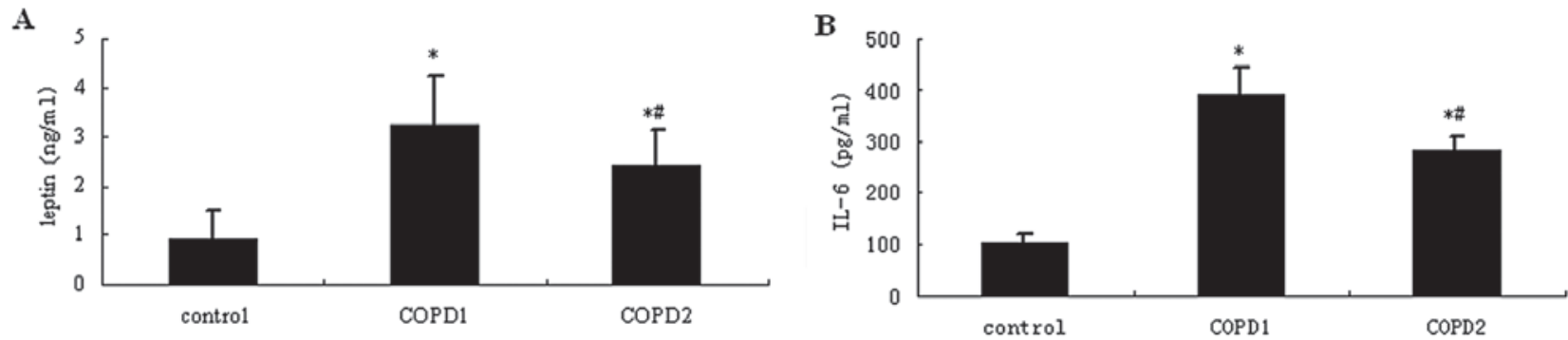

Figure 6. Serum levels of (A) leptin and (B) IL-6 in rats. The results are expressed as the mean \pm standard deviation ( $\mathrm{n}=12$ ). "P<0.05 vs. the control group; ${ }^{\#} \mathrm{P}<0.05$ vs. the COPD1 group. COPD, chronic obstructive pulmonary disease; IL-6, interleukin-6.

were higher in the two COPD groups compared with the control group $(\mathrm{P}<0.05)$. The serum levels of leptin and IL-6 were higher in COPD1 rats compared with those of COPD2 rats $(\mathrm{P}<0.05)$.

\section{Discussion}

COPD is a chronic inflammation of the airways and lungs that is characterized by the infiltration of macrophages, neutrophils and $\mathrm{T}$ lymphocytes. This inflammation may involve a variety of proinflammatory cytokines, including $\mathrm{C}$ reactive protein (CRP), IL-6, IL-8 and tumor necrosis factor- $\alpha(\mathrm{TNF}-\alpha)(11)$. Leptin, which was first identified in 1994 , is a protein hormone encoded by the obese $(o b)$ gene and synthesized in adipose tissues. It is closely associated with energy metabolism and insulin resistance. It mainly regulates body weight by inhibiting appetite and reducing energy intake. Thus, it is important in regulating energy balance, the metabolism of glucose and fat and maintaining the body fat balance. Leptin has also been revealed as a proinflammatory factor (16) and is important in inflammatory responses. Leptin expression has been shown to be increased with infections and inflammation (17), and the leptin receptor has been identified in human alveoli and bronchial epithelial cells (18).

Leptin expression has been identified in human airway epithelial cells and type II alveolar cells. Under physiological conditions, leptin inhibits the appetite, increases energy expenditure and suppresses fat synthesis, which ultimately reduces fat deposition. Under pathological conditions, serum leptin has been suggested to be associated with the initiation and development of certain diseases (19). Leptin is a proinflammatory factor that promotes the release of proinflammatory cytokines (IL-6, IL-1 and TNF- $\alpha$ ) from mononuclear macrophages, and it is regulated by a number of inflammatory factors. Thus, leptin has the characteristics of endocrine hormones and inflammatory cytokines and may interact with other inflammatory cytokines.

During COPD development, obstruction of the peripheral airways, destruction of pulmonary parenchyma and abnormal pulmonary blood vessels reduce the alveolar surface area available for gas exchange, leading to hypoxemia and hypoxia in organs and tissues. Subsequently, cytokines are released, particularly TNF- $\alpha$ and its receptor (TNF- $\alpha$ R) (20). Excessive energy expenditure and a high metabolic rate are partially attributable to increases in respiratory force and oxygen consumption, since the increase in respiratory force is not able to explain body weight loss. Thus, the uncorrected malnutrition that is observed in COPD patients may involve other mechanisms.

Nutrition status is directly associated with the development of COPD and there is evidence showing that malnutrition is an independent risk factor for a poor COPD prognosis. Schols et al (21) showed that the presence of certain cytokines and leptin was the main cause of severe malnutrition and the poor response to nutrition support in COPD patients. In the present study, the clinical investigation and animal experiments demonstrated that the impact of COPD on body weight and nutrition was associated with leptin levels, suggesting that leptin was important in this process.

Bai and $\mathrm{Xu}(22)$ revealed that serum leptin levels were significantly reduced in rats exposed to cigarette smoke and were inversely associated with the amount of smoke exposure. This may have been associated with increased serum catecholamine levels following cigarette smoke exposure, which increases the degradation of fat and leads to a subsequent reduction in leptin via a cAMP-dependent pathway. Additionally, cigarette smoke may alter the sensitivity of leptin receptors in the hypothalamus to regulate leptin synthesis (23). Our study showed that the leptin levels of COPD rats were markedly increased following long-term exposure to cigarette smoke compared with those of the control rats. Furthermore, the rate of body weight gain in these rats increased more slowly than in control rats; in some cases, there was even a body weight loss. This may be attributed to chronic inflammation characterized by the infiltration of macrophages into the alveoli, leading to the production of numerous inflammatory mediators, including TNF- $\alpha$ and IL-6.

After IL-6 enters the circulation, it stimulates the synthesis of leptin by adipose tissues and promotes the synthesis of CRP by hepatocytes (24). IL-6 also facilitates IL-6 synthesis (25) and promotes the degradation of fat tissues. Some studies have suggested that the reduction of leptin levels occurs as a result of a protective response, although the specific mechanisms are yet to be determined.

Our results showed that the levels of leptin and IL-6 in the serum and sputum and the WBC counts of AECOPD patients were higher than those in healthy controls, and these values in the AECOPD patients were increased compared with those of stable COPD patients. Additionally, these variables were inversely related to FEV1\% and ALB levels. These results suggest that leptin levels are associated with the severity of inflammation and that leptin expression increases with 
increased severity. High leptin levels are usually accompanied by a high rate of catabolism and increased energy expenditure, which may result in hypoproteinemia in COPD patients during the acute exacerbation stage.

Furthermore, the expression levels of leptin and IL- 6 in the lungs of the COPD rats were markedly increased, as demonstrated by immunohistochemical analysis and ELISA. These findings suggest that leptin is involved in the occurrence and development of COPD and that it is positively associated with COPD severity. Additionally, the increased leptin expression was accompanied by elevated IL- 6 production, suggesting a correlation between leptin and IL-6.

The interaction between IL-6 and leptin forms a positive feedback loop. IL-6 is primarily secreted by macrophages, lymphocytes and vascular endothelial cells. It has been shown that IL-6 is an important mediator in inflammation and a series of pathophysiological processes, and that it is closely associated with the activity of these diseases (26). IL-6 is a proinflammatory and immune-regulating cytokine that is important in local and systemic inflammatory responses (27). High IL-6 expression may cause damage to vascular endothelial cells, promote immune adhesion and the formation of microthrombi and inhibit the repair of endothelial cells. This results in damage to blood vessels and an increase in their permeability, which may damage the lungs and impair lung function.

IL-6 levels may reflect the severity of inflammation in COPD (28). Walter et al (29) showed that plasma IL-6 levels, together with age and smoking, are independent risk factors for reduced FEV1. Foschino et al (30) demonstrated that IL-6 expression levels in the airway epithelial cells of COPD patients in the acute exacerbation stage were higher compared with those in stable COPD and healthy control patients, and that IL-6 expression levels in stable COPD patients were significantly increased compared with those of healthy controls. These findings are consistent with those of the present study, with the exception that IL-6 levels in patients with stable COPD were only slightly higher compared with those in healthy controls. This suggests that inflammatory mediators and cytokines are maintained at a low level in patients with stable COPD and that leptin and IL-6 are markers of inflammation during acute COPD exacerbations that may be used to evaluate the severity of COPD during the early stage.

The acute recurrence of respiratory infections often leads to COPD development and lung function impairment. LPS is the primary toxic component of endotoxin. It causes direct damage to airway epithelial cells, promotes the chemotaxis of neutrophils and subsequently activates these cells and facilitates the expression of inflammatory cytokines in the bronchi and alveoli. LPS also promotes goblet cell proliferation in the airways, airway remodeling and contraction of the bronchus, which may eventually lead to bronchial inflammation and the development of emphysema within a short period of time.

The investigation of leptin expression in rat COPD models following exposure to LPS and smoke or smoke alone is important for evaluating the association between leptin and COPD. Additionally, the rate of body weight gain in rats exposed to LPS and smoke was slower compared with rats exposed to smoke alone. Pathological examinations indicated that the infiltration of inflammatory cells and emphysema in rats exposed to LPS and smoke were more severe and the expression of leptin and IL-6 were increased compared with rats exposed to smoke alone. This may be due to an increase in leptin following LPS stimulation. COPD induced by exposure to LPS and smoke in animals more closely resembles human COPD.

In COPD patients, the expression levels of leptin and IL-6 were markedly increased and these increases were positively associated with one another. Leptin and IL-6 may form a complex regulatory network to affect the occurrence and development of COPD. Our results also showed that the alveolar wall was negative for IL-6. This suggests that IL-6 is mainly involved in the occurrence of chronic inflammation in the airways.

However, the airways and alveolar walls were positive for leptin. This indicates that leptin may act as an inflammatory mediator in the development of emphysema and promotes the occurrence of local inflammation that is involved in emphysema development. Blood vessel walls were negative for leptin, while the airways and alveolar walls were positive for leptin. It is estimated that leptin levels in the sputum were higher compared with those in the serum; however, these types of samples are not directly comparable.

Our results indicate that levels of leptin and IL-6 in the sputum of AECOPD patients were higher compared with those in stable COPD and healthy control patients, but were similar in stable COPD and healthy control patients. Thus, the detection of leptin and IL-6 levels in the sputum is more clinically important. LPS promotes the expression of leptin and IL-6, suggesting that leptin and IL-6 may serve as biomarkers for airway inflammation in AECOPD patients. Thus, the detection of leptin and IL-6 levels may be important for the determination of COPD severity and prognosis.

\section{References}

1. Vogelmeier C, Hederer B, Glaab T, et al: Tiotropium versus salmeterol for the prevention of exacerbations of COPD. N Engl J Med 364: 1093-1103, 2011.

2. Calverley PM, Anderson JA, Celli B, et al: Salmeterol and fluticasone propionate and survival in chronic obstructive pulmonary disease. N Engl J Med 356: 775-789, 2007.

3. Struik FM, Duiverman ML, Bladder G and Wijkstra PJ: Effects of non-invasive positive pressure ventilation (NIPPV) in stable chronic obstructive pulmonary disease (COPD). Respir Med COPD update 4: 94-100, 2008.

4. Murray CJ and Lopez AD: Alternative projections of mortality and disability by cause 1990-2020: Global Burden of Disease Study. Lancet 349: 1498-1504, 1997.

5. Fang $X$, Wang $X$ and Bai C: COPD in China: the burden and importance of proper management. Chest 139: 920-929, 2011.

6. Yoon HI and Sin DD: Confronting the colossal crisis of COPD in China. Chest 139: 735-736, 2011.

7. Siafakas NM, Vermeire P, Pride NB, et al: Optimal assessment and management of chronic obstructive pulmonary disease (COPD). The European Respiratory Society Task Force. Eur Respir J 8: 1398-1420, 1995.

8. de Boer WI, Sont JK, van Schadewijk A, Stolk J, van Krieken JH and Hiemstra PS: Monocyte chemoattractant protein 1, interleukin 8 , and chronic airways inflammation in COPD. J Pathol 190: 619-626, 2000.

9. Lewis MI and Belman MJ: Nutrition and the respiratory muscles. Clin Chest Med 9: 337-348, 1988.

10. Nishimura $Y$, Tsutsumi M, Nakata $H$, Tsunenari T, Maeda $H$ and Yokoyama M: Relationship between respiratory muscle strength and lean body mass in men with COPD. Chest 107: 1232-1236, 1995.

11. Wouters EF, Groenewegen KH, Dentener MA and Vernooy JH: Systemic inflammation in chronic obstructive pulmonary disease: the role of exacerbations. Proc Am Thorac Soc 4: 626-634, 2007. 
12. Broekhuizen R, Vernooy JH, Schols AM, Dentener MA and Wouters EF: Leptin as local inflammatory marker in COPD. Respir Med 99: 70-74, 2005.

13. Auwerx J and Staels B: Leptin. Lancet 351: 737-742, 1998.

14. Yang YM, Sun TY and Liu XM: The role of serum leptin and tumor necrosis factor-alpha in malnutrition of male chronic obstructive pulmonary disease patients. Chin Med J (Engl) 119: 628-633, 2006

15. Lam QL and Lu L: Role of leptin in immunity. Cell Mol Immunol 4: 1-13, 2007.

16. Bellmeyer A, Martino JM, Chandel NS, et al: Leptin resistance protects mice from hyperoxia-induced acute lung injury. Am J Respir Crit Care Med 175: 587-594, 2007.

17. Otero M, Lago R, Lago F, et al: Leptin, from fat to inflammation: old questions and new insights. FEBS Lett 579: 295-301, 2005.

18. Vernooy JH, Drummen NE, van Suylen RJ, et al: Enhanced pulmonary leptin expression in patients with severe COPD and asymptomatic smokers. Thorax 64: 26-32, 2009.

19. Meier U and Gressner AM: Endocrine regulation of energy metabolism: review of pathobiochemical and clinical chemical aspects of leptin, ghrelin, adiponectin, and resistin. Clin Chem 50: 1511-1525, 2004.

20. Sevenoaks MJ and Stockley RA: Chronic Obstructive Pulmonary Disease, inflammation and co-morbidity - a common inflammatory phenotype? Respir Res 7: 70, 2006.

21. Schols AM, Creutzberg EC, Buurman WA, et al: Plasma leptin is related to proinflammatory status and dietary intake in patients with chronic obstructive pulmonary disease. Am J Respir Crit Care Med 160: 1220-1226, 1999.
22. Bai XL and Xu JY: Smoking to rat blood lean meat, adiponectin, IL-6 and c-reactive protein influence. Chin J Respir Crit Care Med 9: 298-299, 2010 (In Chinese).

23. Sull JW, Kim HJ, Yun JE, et al: Serum adiponectin is associated with smoking status in healthy Korean men. Endocr J 56: 73-78, 2009.

24. Trujillo ME, Sullivan S, Harten I, et al: Interleukin-6 regulates human adipose tissue lipid metabolism and leptin production in vitro. J Clin Endocrinol Metab 89: 5577-5582, 2004.

25. Fantuzzi G: Adipose tissue, adipokines, and inflammation. J Allergy Clin Immunol 115: 911-919, quiz 920, 2005.

26. Rose-John S, Waetzig GH, Scheller J, et al: The IL-6/sIL-6R complex as a novel target for therapeutic approaches. Expert Opin Ther Targets 11: 613-624, 2007.

27. Yanbaeva DG, Dentener MA, Creutzberg EC and Wouters EF: Systemic inflammation in COPD: is genetic susceptibility a key factor? COPD 3: 51-61, 2006.

28. Wouters EF: Local and systemic inflammation in chronic obstructive pulmonary disease. Proc Am Thorac Soc 2: 26-33, 2005.

29. Walter RE, Wilk JB, Larson MG, et al: Systemic inflammation and COPD: the Framingham Heart Study. Chest 133: 19-25, 2008.

30. Foschino Barbaro MP, Carpagnano GE, Spanevello A, et al: Inflammation, oxidative stress and systemic effects in mild chronic obstructive pulmonary disease. Int J Immunopathol Pharmacol 20: 753-763, 2007. 